\title{
Erratum
}

Lixia Guo, Song Li, Ling Zhong*, Lei Guo, and Lunyan Wang

\section{Inverse analysis of concrete meso-constitutive model parameters considering aggregate size effect}

https://doi.org/10.1515/secm-2021-0033

received May 25, 2020; accepted September 15, 2020

Erratum to: Lixia Guo, Song Li, Ling Zhong, Lei Guo, and Lunyan Wang. Inverse analysis of concrete meso-constitutive model parameters considering aggregate size effect. Sci Eng Compos Mater. 2020;27(1):397-404. doi: 10.1515/ secm-2020-0043.

The authors would like to change the order of Lixia Guo's affiliation in "Inverse analysis of concrete meso-constitutive model parameters considering aggregate size effect," 2020;27(1):397-404.

\footnotetext{
* Corresponding author: Ling Zhong, School of Water Conservancy, North China University of Water Resources and Electric Power, Zhengzhou, 450046, China; Henan Key Laboratory of Water Environment Simulation and Treatment, Zhengzhou, 450046, China; Henan Water Valley Research Institute, Zhengzhou, 450046, China, e-mail: 250514782@qq.com

Lixia Guo: School of Water Conservancy, North China University of Water Resources and Electric Power, Zhengzhou, 450046, China; Henan Key Laboratory of Water Environment Simulation and Treatment, Zhengzhou, 450046, China; Henan Water Valley Research Institute, Zhengzhou, 450046, China; Research Center on Levee Safety and Disaster Prevention, Ministry of Water Resources, Zhengzhou, 450003, China

Song Li: School of Water Conservancy, North China University of Water Resources and Electric Power, Zhengzhou, 450046, China Lei Guo, Lunyan Wang: School of Water Conservancy, North China University of Water Resources and Electric Power, Zhengzhou, 450046, China; Henan Key Laboratory of Water Environment Simulation and Treatment, Zhengzhou, 450046, China; Henan Water Valley Research Institute, Zhengzhou, 450046, China
}

\section{Author information}

Lixia Guo: School of Water Conservancy, North China University of Water Resources and Electric Power, Zhengzhou, 450046, China; Henan Key Laboratory of Water Environment Simulation and Treatment, Zhengzhou, 450046, China; Henan Water Valley Research Institute, Zhengzhou, 450046, China; Research Center on Levee Safety and Disaster Prevention, Ministry of Water Resources, Zhengzhou, 450003, China. 